\title{
Transient 23-30 Hz Oscillations in Mouse Hippocampus During Exploration of Novel Environments
}

\author{
Joshua D. Berke, ${ }^{1,2 *}$ Vaughn Hetrick, ${ }^{1}$ Jason Breck, ${ }^{1}$ and Robert W. Greene ${ }^{3,4}$
}

\begin{abstract}
The hippocampus is a key brain structure for the encoding of new experiences and environments. Hippocampal activity shows distinct oscillatory patterns, but the relationships between oscillations and memory are not well understood. Here we describe bursts of hippocampal $\sim 23-30 \mathrm{~Hz}$ (beta2) oscillations in mice exploring novel, but not familiar, environments. In marked contrast to the relatively invariant $\sim 8$ $\mathrm{Hz}$ theta rhythm, beta2 power was weak during the very first lap of the novel environment, increased sharply as the mice reencountered their start point, then persisted for only a few minutes. Novelty-evoked oscillations reflected precise synchronization of individual neurons, and participating pyramidal cells showed a selective enhancement of spatial specificity. Through focal viral manipulations, we found that novelty-evoked oscillations required functional NMDA receptors in CA3, a subregion critical for fast oscillations in vitro. These findings suggest that beta2 oscillations indicate a hippocampal dynamic state that facilitates the formation of unique contextual representations. $\odot 2008$ Wiley-Liss, Inc.
\end{abstract}

KEY WORDS: oscillations; interneurons; novelty; mouse; NMDA receptor

\section{INTRODUCTION}

The hippocampus plays critical roles in episodic memory, spatial/contextual processing, and novelty detection (Knight, 1996; Eichenbaum and Cohen, 2001). A primary goal of hippocampus research is to understand how neurons and neural circuits are able to achieve these behavioral functions. Pyramidal cells in hippocampal areas CA1 and CA3 are selectively active as animals visit particular parts of a given environment ("place cells"), and such neurons may be components of spatial/contextual representations. Upon exposure to a novel environment, place-selective firing develops within seconds to minutes (Wilson and McNaughton, 1993; Frank et al., 2004), but then can remain stable for months (Thompson and Best, 1990). Although it has been shown that NMDA receptors in the CA3 subregion are critical for the rapid development of place cell spatial specificity (Nakazawa et al., 2003), it is not known how the hippocampus is able to combine rapid plasticity with long-term stability (the "stability-plasticity dilemma"; Grossberg, 1999).

\footnotetext{
${ }^{1}$ Department of Psychology, University of Michigan, Ann Arbor, Michigan; ${ }^{2}$ Program in Neuroscience, University of Michigan, Ann Arbor, Michigan; ${ }^{3}$ Department of Psychiatry, University of Texas Southwestern, Dallas, Texas; ${ }^{4}$ Department of Psychiatry, Dallas VAMC, Dallas, Texas.

This article includes supplementary video, available online at http:// www.interscience.wiley.com/jpages/1050-9631/suppmat.

Grant sponsors: Whitehall Foundation; The National Institute on Drug Abuse; National Institute of Mental Heath; The Department of Veterans Affairs.

*Correspondence to: Joshua Berke, Department of Psychology, University of Michigan, Ann Arbor, MI 48104, USA. E-mail: jdberke@umich.edu Accepted for publication 22 February 2008

DOI 10.1002/hipo.20435

Published online 8 April 2008 in Wiley InterScience (www. interscience.wiley.com).
}

In artificial neural networks, this problem is typically solved by allowing or blocking plasticity in distinct epochs of encoding and retrieval, and similarly, it has been suggested that hippocampal plasticity is sharply enabled or reduced under distinct functional states (Hasselmo, 1999; Meeter et al., 2004). Obvious transitions between hippocampal states can be detected in the rhythmic firing patterns of single cells, and in local field potentials (LFP; Buzsáki, 2006). For example, in awake, moving animals hippocampal activity shows strong theta $(\sim 5-11 \mathrm{~Hz})$ and gamma $(\sim 35-100 \mathrm{~Hz})$ oscillations, while during slow-wave sleep and quiet rest the hippocampus shows irregular activity marked by sharp waves and ripples $(\sim 140-200 \mathrm{~Hz})$. However, while it has been suggested that ripples may play a role in the consolidation of recent experiences (Foster and Wilson, 2006; Diba and Buzsaki, 2007), little is known about the relationships between electrophysiological patterns and the on-line encoding of memories (Hasselmo et al., 2002).

\section{RESULTS}

Novel Environments Evoke Transient 23- to $30-\mathrm{Hz}$ Oscillations

We recorded from hippocampal regions CA1 and CA3 of mice exploring novel environments. In the example in Figure 1, the mouse was placed onto a novel enclosed rectangular track and immediately began running laps. During the initial 1-2 min of exploration, we observed strong pulses of LFP oscillations in the 23-30 $\mathrm{Hz}$ (beta2) range [Figs. 1(b,c); see also Supplementary Video Online]. Beta2 power was low after the first 2 min, even though the animal continued to run laps and theta power remained high. Upon reintroduction to the same environment the next day, theta power was again high, but beta 2 power was much lower than during the initial exposure to that environment.

To confirm that beta2 power was selectively enhanced during the early exploration of new places, mice were exposed to a 3-day sequence of novel and familiar environments (Fig. 2). Although mice engaged in spontaneous exploration during all sessions, they were able to distinguish novel and familiar environments, as shown by reductions in exploration time (proportion of time running in first $10 \mathrm{~min}$, mean \pm SEM: novel, $79.3 \pm 8.0 \%$; familiar, $55.9 \pm$ 
a

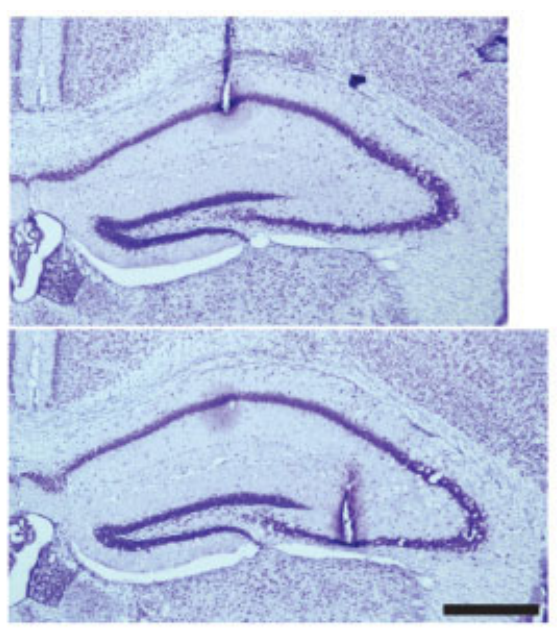

b

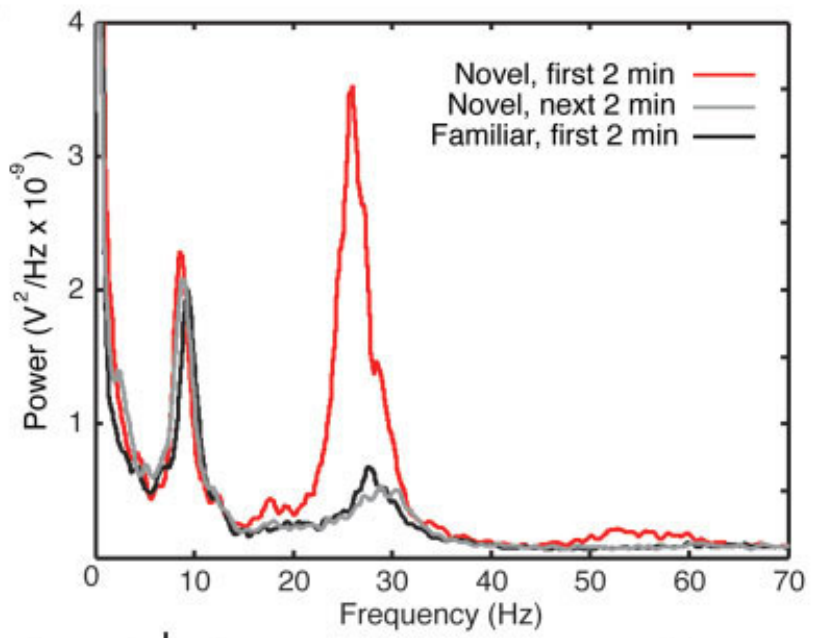

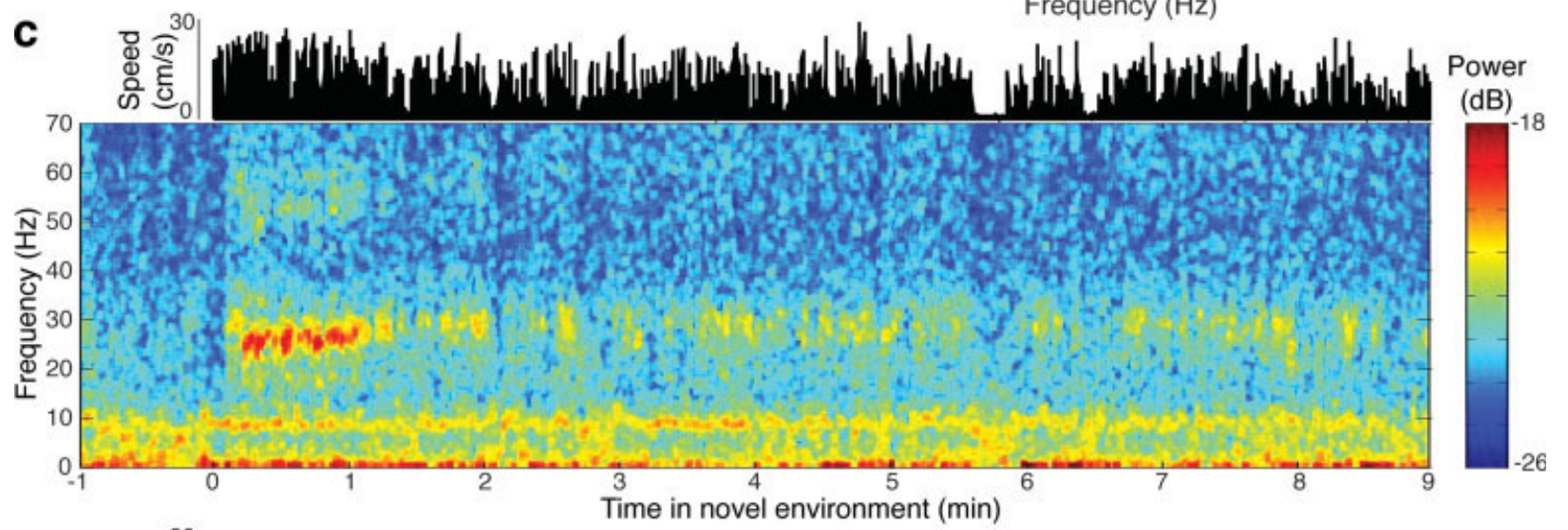

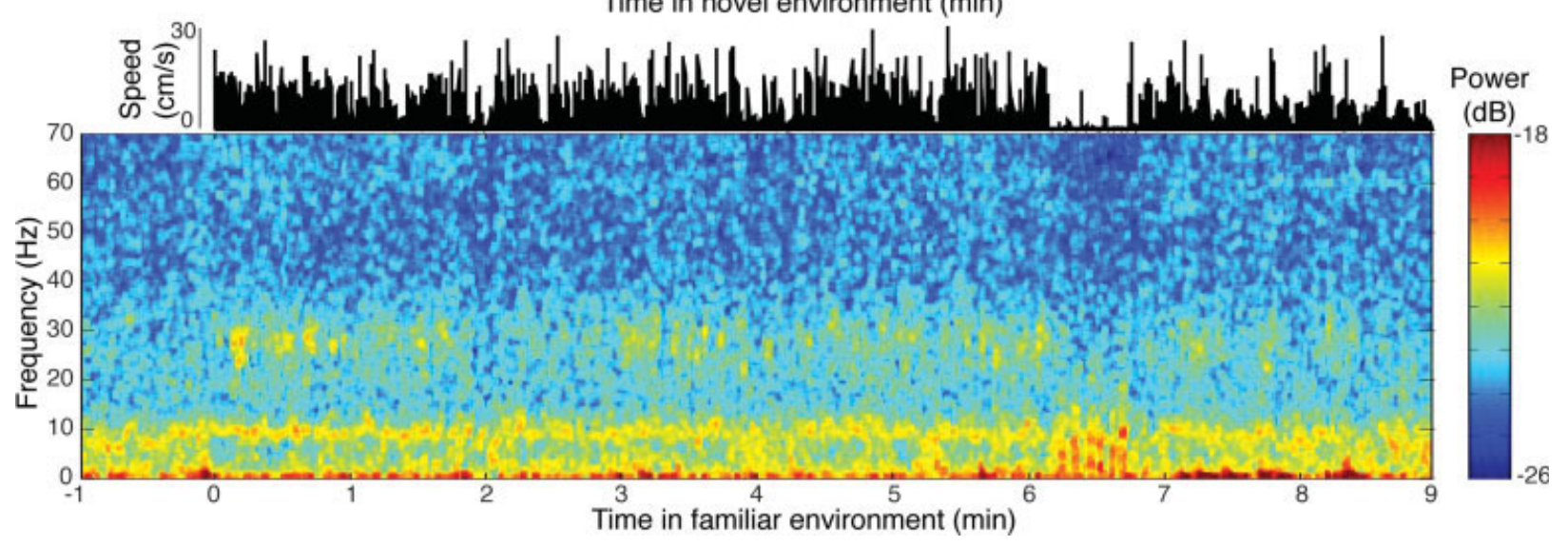

FIGURE 1. Transient 23-30 Hz (beta2) oscillations during exploration of a novel environment. a, Simultaneous tetrode recording locations in CA1 (top) and CA3 (bottom) pyramidal layers. Marker lesions were produced by passing current through the tetrode after recordings are completed. Scale bar, $0.5 \mathrm{~mm}$. b, Power spectral density of LFP from the CA3 location shown in (a). During the first 2 min of exploring a novel enclosed track, power is high in both theta $(\sim 8 \mathrm{~Hz})$ and beta2 bands. During the following $2 \mathrm{~min}$, beta2 power is much lower but theta power remains high. Beta2 power remains low during the first 2 min in the same environment the next day. c, Time courses of locomotor activity and spectral power comparing the novel environment (upper) to the same environment repeated the next day (lower). In both instances the mouse showed near-continuous motion until pausing after $\sim 6 \mathrm{~min}$.
3.9\%). Analysis of variance (ANOVA) showed that beta2 power significantly changed across time $(P<0.001)$ and session $(P<$ 0.001 ), and planned comparisons showed that beta 2 power in the first 2 min was significantly enhanced in novel compared to familiar environments (CA3, $t=4.01, P=0.001$; CA1, $t=3.02, P=0.005)$. By contrast, theta in the first 2 min was not different between novel and familiar environments (CA3, $t=0.94, P=0.36$; CA1, $t=-0.19, P=0.85$ ).

Although beta/gamma oscillations are prominent in olfactory structures (Adrian, 1942; Ravel et al., 2003), placing mice in a 

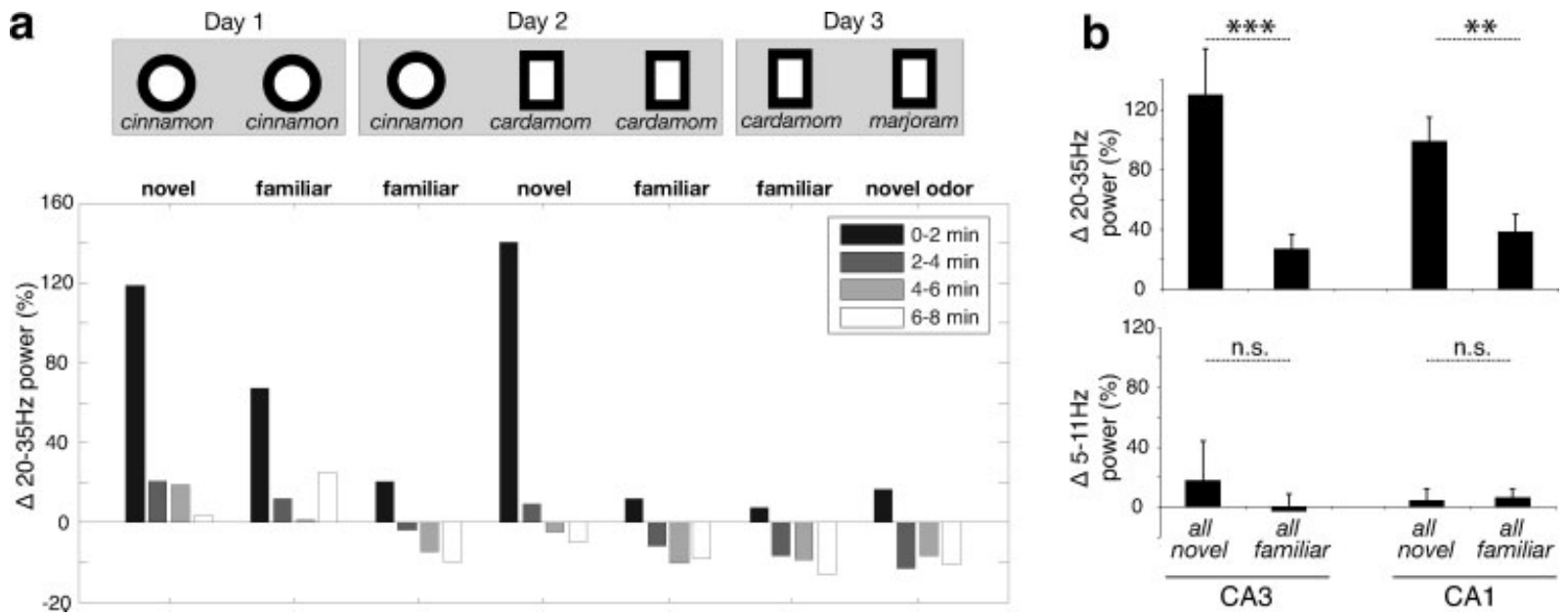

FIGURE 2. Novel environments transiently enhance beta2 but not theta oscillations. a, (Top) Example 3-day sequence of novel and familiar environments. (Bottom) Change in CA3 beta2 power, calculated in 2-min blocks from the start of entry into each environment, for 5 mice ( 3 mice with no viral injection, 2 mice with
AAV-LacZ into CA3). b, Changes in beta2 (top) and theta (bottom) power during the first 2 min block, comparing all novel to familiar environments for both CA3 and CA1 (same 5 mice as in a). ${ }^{* * *}, \boldsymbol{P}<0.001$; ${ }^{* *}, \boldsymbol{P}<0.005$; n.s., not significant $(\boldsymbol{P}>\mathbf{0 . 1})$. familiar environment with an additional novel odor was not sufficient to enhance hippocampal beta2 oscillations (planned comparison to familiar environments, CA3, $t=-0.431, P=$ 0.67; CA1, $t=0.38, P=0.56$ ) and behaviorally the micetreated such environments as familiar (proportion of time running $=53.2 \pm 11 \%)$.

\section{Novelty-Evoked Oscillations Are Weak in the Very First Lap}

High beta2 power was selectively observed when mice were actively moving through the novel environments (Fig. 3). However, unlike the theta rhythm, beta2 oscillations did not commence as soon as the mice began running in the novel environment. For the mouse illustrated in Figure 1, beta2 power was very low during the first lap, and only reached full strength as the mouse began the third lap, despite comparable running speed during the first three laps (Fig. 4a). Although the time taken for mice to complete the first lap varied from $7 \mathrm{~s}$ to over $40 \mathrm{~s}$, the onset of strong beta 2 was consistently delayed until the first lap was complete (Fig. 4).

\section{Precise Entrainment of Hippocampal Neurons}

We examined the timing of spikes of 126 individual hippocampal neurons from three mice, relative to the troughs of novelty-evoked beta2 oscillations (Fig. 5). Fast-spiking interneurons are especially important for the intrahippocampal generation of fast rhythms (Fuchs et al., 2007), and we observed that, in all mice, presumed fast-spiking interneurons were strongly entrained to beta2 oscillations (3/3 in CA3, one from each mouse, and $3 / 4$ in CA1, using significance threshold of $P<$
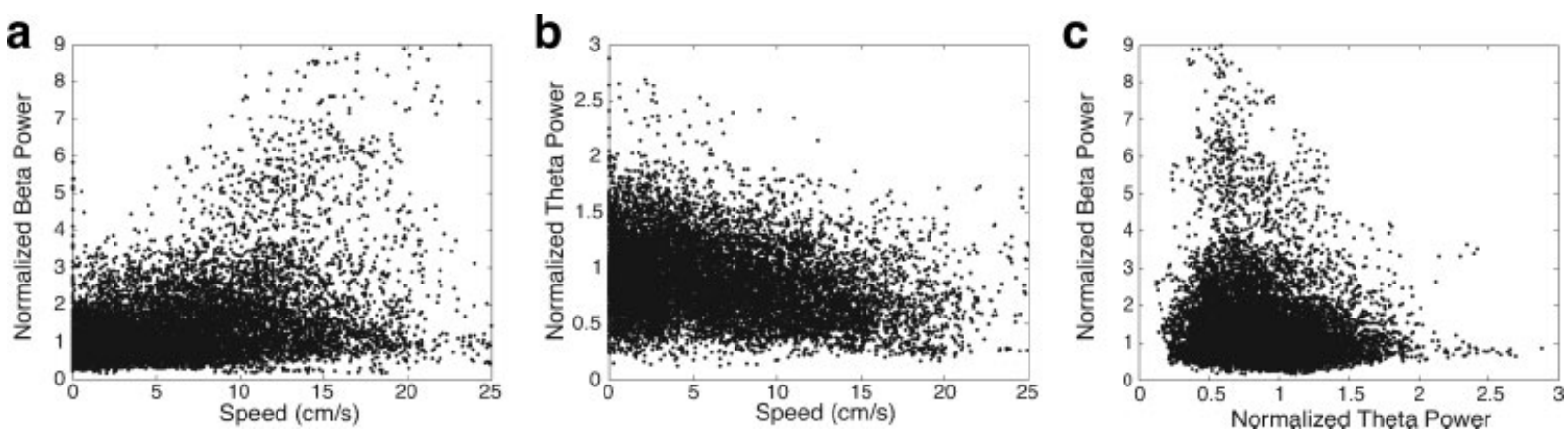

FIGURE 3. Hippocampal beta2 oscillations occur during active movement through the novel environment. All points shown are from the first 2 min of novel sessions only. Beta2 $(20-35 \mathrm{~Hz})$ and theta $(5-11 \mathrm{~Hz})$ power in $1 \mathrm{~s}$ epochs was measured by wavelet convolution and normalized to their respective mean values during the first $10 \mathrm{~min}$ of the novel sessions, averaging CA3, CA1 to- gether. a, Beta2 power vs. speed. Note cloud of points of high beta 2 power occurs at moderate to high speed values. b, Theta power vs. speed. c, Beta2 power vs. theta power. Note that few points occur along the main diagonal. High beta2 power often occurs when theta power is low, suggesting that beta 2 may be replacing rather than coexisting with theta. 
a lap 1 lap 2 lap 3
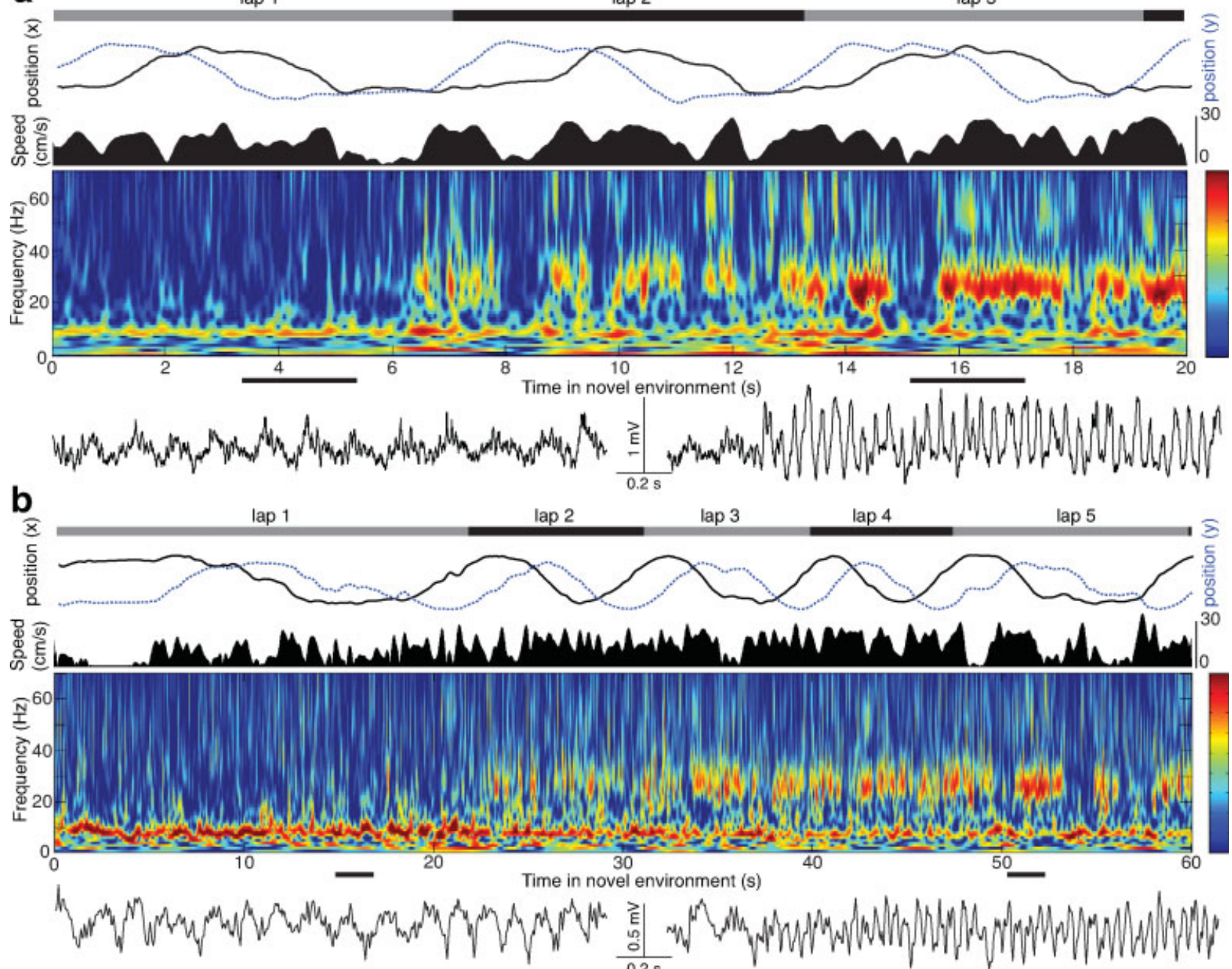

lap 3 lap 4

lap 5

C

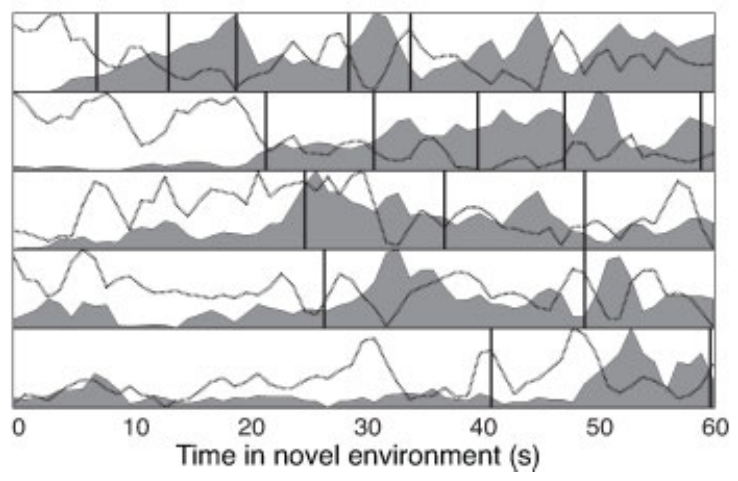

FIGURE 4. Novelty-evoked beta2 oscillations are delayed until after the first lap, while theta oscillations are not. a, Same example as Figure 1, on finer time scale (see also Supplementary Video Online). Upper panels indicate lap count, position and speed during initial exploration. Middle colored panel shows wavelet scalogram for CA3 LFP (complex Morlet wavelet). Note that beta2 power is low during the first lap of this rectangular environment, then moderate for the second lap, becoming very strong as the mouse begins the third lap. By contrast, theta power is strong from the very start. Bottom panel shows raw CA3 LFP for each of the 2-s epochs marked by solids black bars in the middle panel. $b$, Another example of delayed onset of beta2 oscillations, from a different mouse exploring the novel circular environment (this mouse was injected with AAV-LacZ virus; see text). Layout is the same as a, but note different time scales. Although it took this mouse $>3$ times as long to complete the first lap, beta2 power was still delayed until lap2. c, Examples of CA1 LFP power time courses in novel environments (each row shows an example from a different mouse; top example is same as Figs. 1, 4a). Vertical lines indicate lap completions (for the first 5 laps only). Solid gray area indicates beta2 $(20-35 \mathrm{~Hz})$ power, dashed line indicates theta $(5-11 \mathrm{~Hz})$ power, in both cases normalized to the maximum instantaneous power over the first minute. Note that peak beta 2 power is consistently delayed until after the completion of the first lap, and that beta2 and theta are not closely coupled. d, Lap-by-lap power in novel environments, comparing CA1 beta 2 to theta. $n=8$ (5 mice $\times 2$ novel sessions, excluding 2 sessions in which the mice reversed direction before running five laps). Power values are normalized to the maximum lap power over the first five laps. The difference between normalized mean beta 2 and mean theta was significant during the first lap (***, paired $t=-9.62, P<0.001$ ), but not for subsequent laps $(P>0.05$ after correcting for multiple comparisons). 

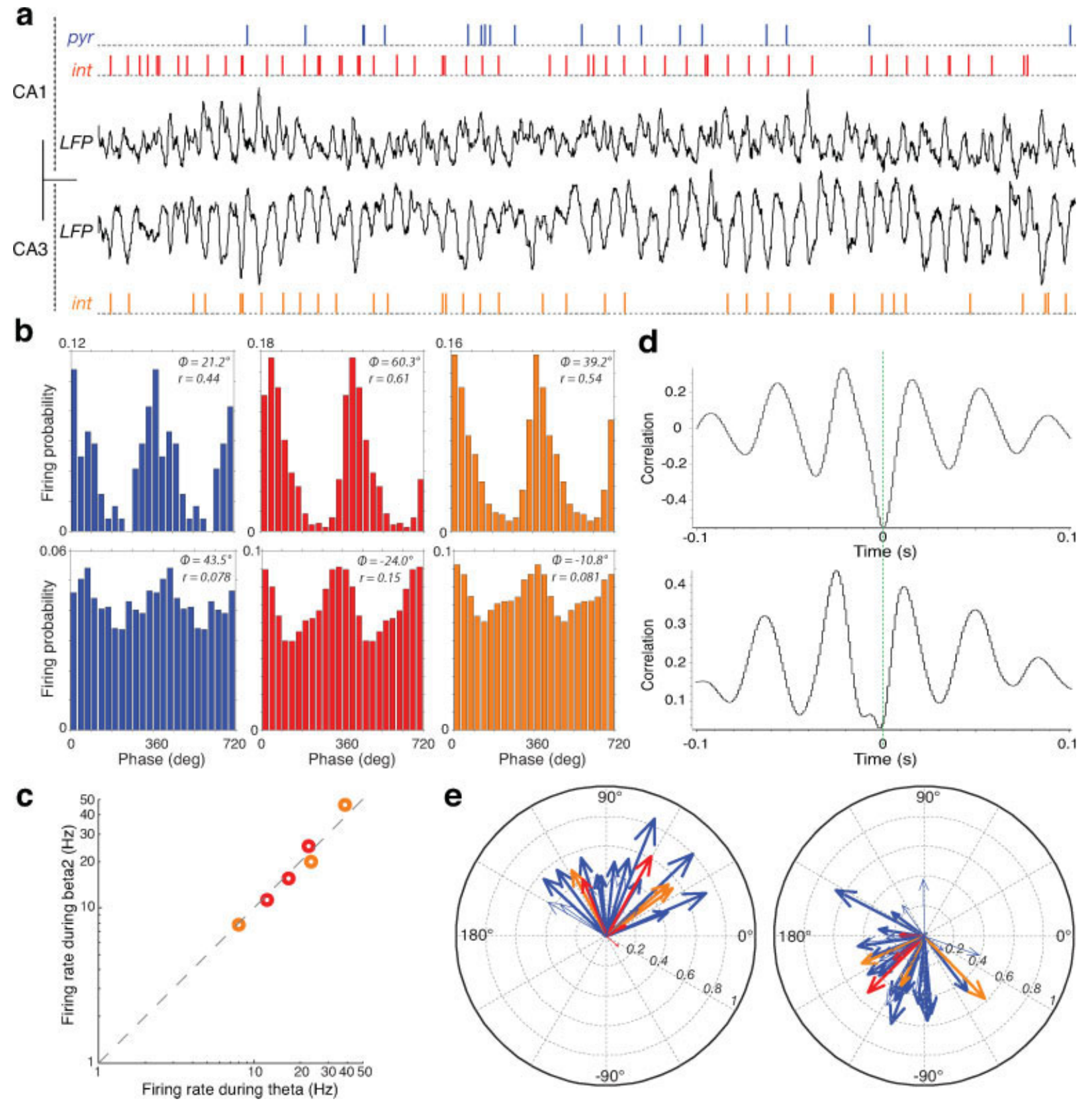

FIGURE 5. Entrainment of hippocampal CA3 and CA1 neurons to novelty-evoked beta oscillations. a, Examples of simultaneously recorded LFP and single-unit spike times for a 2 -s period including novelty-induced beta2. pyr, int indicate presumed pyramidal cell and fast-spiking interneurons. $b$, Phase histograms for the three neurons indicated in a. In the upper panels, $0 \% 360^{\circ} \%$ $720^{\circ}$ indicates troughs of CA3 beta 2 oscillations (25.3s of CA3 beta 2 was detected). The significance of beta2-entrainment for the three neurons was (left to right) $P=2.31 \times 10^{-6}, 2.08 \times 10^{-86}$, $2.12 \times 10^{-56}$. In the lower panels, $0 \% 360^{\circ} / 720^{\circ}$ indicates troughs of CA1 theta oscillations (1981s of theta was detected). Thetaentrainment phases were consistent with previous observations in the mouse (Buzsaki et al., 2003). $\Phi, r$ indicate phase angle and magnitude of the mean spike vector. c, Entrainment of fast-spiking interneurons to beta2 oscillations occurs without substantial change in firing rate. Each circle indicates the firing rates for one cell, comparing detected beta 2 intervals vs. detected theta (but not beta2) intervals, during the first $10 \mathrm{~min}$ of exposure to a novel environment (same data set as Fig. 3). Red, CA1 presumed fast-

spiking interneurons; orange, CA3 presumed fast-spiking interneurons. Dashed line indicates equal firing rate during theta and beta2. d, CA3 and CA1 beta 2 oscillations are out of phase. Crosscorrelations between LFPs recorded from CA3 and CA1 pyramidal layers, during detected epochs of novelty-evoked beta2 at the CA3 site (CA3 is the correlation reference). Top plot uses LFPs from the two sites shown in Figure 1, bottom plot is from another mouse in which the CA1, CA3 locations were similar (i.e., CA3b/c pyramidal layer). Note oscillation at beta 2 frequency, with lower correlation at time zero indicating that the rhythms are out of phase in the two subregions. e, Entrainment of neuronal populations to beta 2 oscillations in CA3 (left) or CA1 (right). Polar plots of $\Phi, \mathbf{r}$ for all neurons with $\geq 10$ spikes during the corresponding detected beta 2 oscillations. Thicker arrows indicate cells that were significantly entrained $(P<0.01)$. Blue arrows, CA1/CA2 pyramidal cells; red arrows, CA1 fast-spiking interneurons; orange arrows, CA3 fast-spiking interneurons. CA1/CA2 pyramidal neurons and fast-spiking interneurons in both CA1 and CA3 were active on the ascending phase of CA3 beta2/descending phase of CA1 beta2. 
0.01). The entrainment of hippocampal interneurons to beta2 pulses occurred without a substantial change in their firing rate, compared to their firing rate during theta (Fig. 5c), and these cells also showed normal patterns of theta-entrainment over the course of the whole session. However, the precision of beta2entrainment was much higher than theta-entrainment (Fig. 5b).

Although many pyramidal neurons, especially in CA3, did not fire sufficient spikes during detected beta2 pulses to accurately assess entrainment, the majority of CA1/CA2 pyramidal neurons that fired at least 10 spikes during either CA1 and/or CA3 beta2 were significantly entrained (22/37 with a significance cutoff of $P<0.01$, or $26 / 37$ at $P<0.05$ ). Both pyramidal cells and fast-spiking interneurons fired with similar, consistent phase (Fig. 5e), indicating that the observed beta2 rhythm reflects the highly synchronous activity of neuronal populations within narrow temporal windows $(\sim 1 / 4$ cycle, corresponding to $\sim 10 \mu \mathrm{s}$ ).

\section{Entrained Pyramidal Cells Gain Spatial Specificity}

All beta2-entrained pyramidal neurons developed place-specific firing during exploration (e.g. Fig. 6a). We examined the time course of this process using two established measures (Skaggs et al., 1993; Jung et al., 1994), spatial information and sparsity [Figs. 6(b,c); lower sparsity indicates greater spatial specificity]. Pyramidal cells that were persistently active in the novel environment were divided into two groups-those for which beta2-entrainment reached significance (at $P<0.01$ ) and the rest. For both measures of spatial specificity, ANOVA indicated an interaction between cell entrainment and time (spatial information, $P=0.015$; sparsity, $P=0.033$ ). Beta2-entrained cells had lower spatial specificity than nonentrained cells during the first minute (planned comparison Mann-Whitney tests: sparsity, $P=0.034$; spatial information $P=0.034$ ), but showed a sharp increase in spatial specificity between minutes 1 and 2 while beta oscillations were occurring (planned comparison Wilcoxon signed ranks tests: sparsity, $P=0.038$; spatial information, $P=$ 0.028). No such change was seen for nonentrained cells (Wilcoxon tests; sparsity, $P=0.917$; spatial information, $P=$ 0.575). In one of the mice we were able to record many CA1 place cells simultaneously, and we found that the extent of betaentrainment predicted the improvement in spatial specificity between the first $2 \mathrm{~min}$ (Fig. 6d). These results are consistent with a model in which a subpopulation of CA1 hippocampal neurons display place-selectivity immediately (Frank et al., 2004), most likely because they receive appropriate connections from entorhinal cortex, while the others rapidly acquire spatial selectivity while participating in beta 2 oscillations.

\section{CA3 NMDA Receptors Are Required for Novelty-Evoked Oscillations}

The CA3 region is particularly important in the generation of fast hippocampal rhythms, in vitro (Shimono et al., 2000; Arai and Natsume, 2006), and in vivo (Csicsvari et al., 2003).
NMDA receptors on CA3 cells are required when contextual representations are rapidly encoded or reorganized (Lee and Kesner, 2002; Nakazawa et al., 2003), and are essential for the within-session development of place-specific firing of CA1 pyramidal cells in novel environments (Nakazawa et al., 2003). We therefore investigated whether CA3 NMDA receptors are involved in the novelty-evoked generation of beta2 oscillations. We employed a combined viral/genetic strategy (Scammell et al., 2003) to achieve an anatomically and temporally constrained removal of NMDA receptors. This involved unilateral infusion of an adenoassociated virus vector for Cre recombinase (AAV-Cre), into our mice, in which the gene for the NMDA receptor essential subunit NR1 was "floxed" ( $A N R 1$ mice). This approach, which minimizes potential developmental effects of the gene deletion, has been shown to cause a selective, functional removal of NMDAr-mediated synaptic transmission and plasticity, and a behavioral deficit in new learning in a paired associate task (Rajji et al., 2006). We observed that infusion of AAV-Cre into CA3 completely blocked the novelty-enhancement of beta2 oscillations (Fig. 7), without apparent effect on the power of the theta rhythm (data not shown). By contrast, mice that received the control virus AAV-LacZ showed normal enhancement of beta2 oscillations in the novel environments (e.g., Fig. 4b). ANOVA showed a significant interaction between novelty and viral treatment $(P<0.001)$, but no three-way interaction with location (CA3 vs. CA1; $P=0.55$ ). Both controls and AAV-Cre mice did show a slight increase in beta2 power upon entry into familiar environments, but for AAV-Cre mice this was of similar magnitude for novel and familiar environments (Fig. 7b).

\section{DISCUSSION}

In exploring mice, we found a specific neural activity difference between novel and familiar environments-the power of hippocampal 23- to $30-\mathrm{Hz}$ (beta2) oscillations. This difference was not observed for the hippocampal theta rhythm, and cannot readily be accounted for by overt behavioral differences such as running speed. Both pyramidal cell and interneuron populations showed tight phase-locking to the beta2 rhythm, and participating pyramidal cells selectively gained spatial specificity during beta2. Novelty-evoked oscillations required functional NMDA receptors in CA3, consistent with previous reports that this receptor population is critical for the rapid development of spatial specificity.

In rats and rabbits, rhythmic activity in the awake hippocampus has been studied for decades (Buzsáki, 2006), so it is perhaps surprising that the strong beta 2 oscillations we observed in novel environments have not been commonly reported. A previous characterization of mouse hippocampal rhythms (Buzsaki et al., 2003) found gamma oscillations, but these were of higher frequency (40-100 Hz, centered around $60 \mathrm{~Hz}$ ) and were tightly coupled to the theta rhythm. [This theta-linked, higher frequency gamma rhythm was often visible in our data too, for example in the left- 


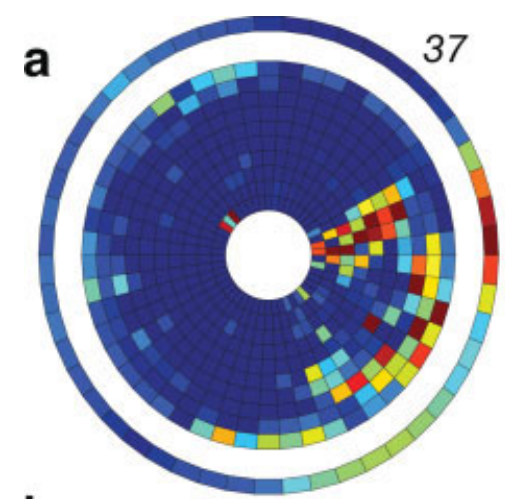

b

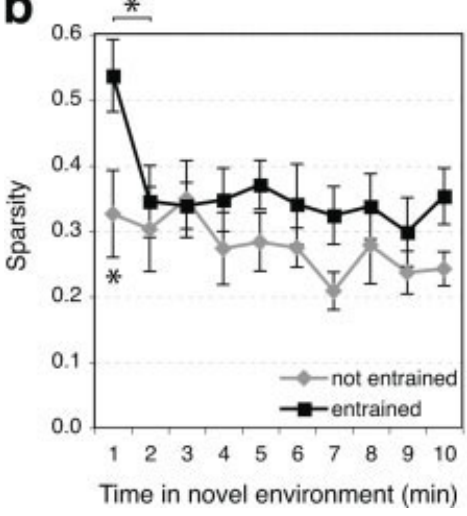

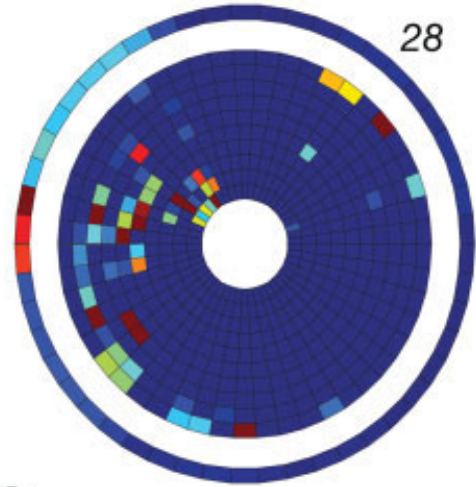

C

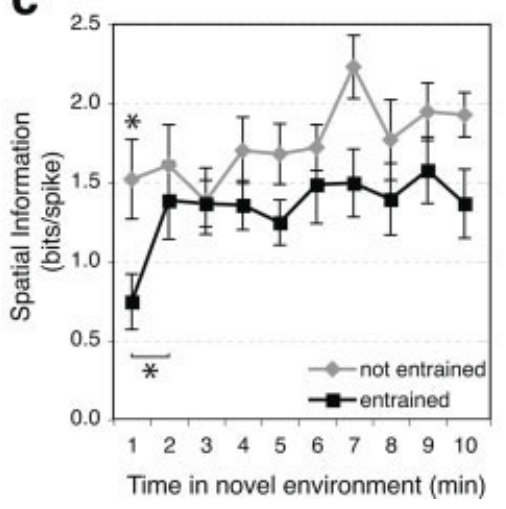

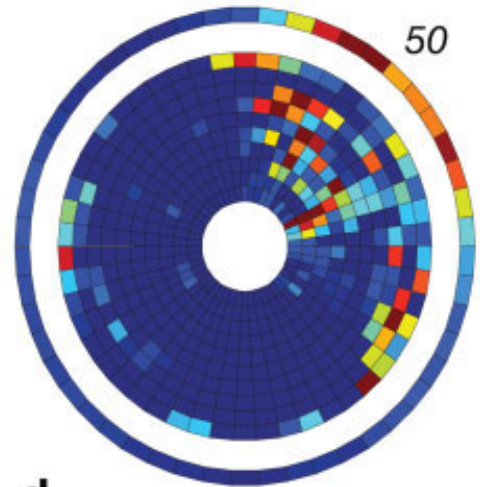

d

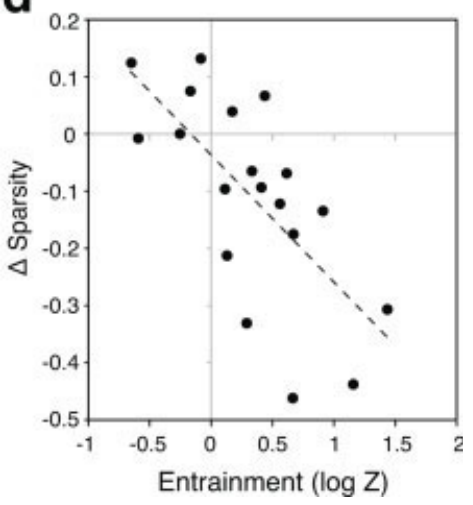

FIGURE 6. Beta2-entrained cells increase spatial selectivity during the first $2 \mathrm{~min}$. a, Representative examples of spatiallyselective firing of beta2-entrained CA1 pyramidal cells. For each plot, the separate outer ring indicates overall firing in 50 angular position bins, during the first $10 \mathrm{~min}$ of exposure to a novel environment; the number to the top right indicates the color scale maximum (in $\mathrm{Hz}$ ). Each of the inner rings shows a 1-min time block (earliest is outermost), with the color scale normalized to the maximum firing rate in that minute. b, c, Two measures of the spatial specificity of CA1/CA2 pyramidal cells over the first 10 min of exploring a novel environment. To enable calculation of specificity measures across time, only cells that fired more than 12 spikes in every minute block were included $(n=9$ entrained, $n=8$ nonentrained; values are mean \pm SEM). Sparsity values can range from 0 to 1 , with lower values indicating higher specificity (see Methods). Significantly beta2-entrained cells initially have lower spatial specificity, but show an improvement in spatial specificity between minutes 1 and 2; nonentrained cells do not (asterisks, $P<0.05)$. d, Within-animal examination of the relationship between beta 2 entrainment and increase in spatial specificity. Rayleigh's $Z$ is a measure of the significance of entrainment. $Y$-axis shows change in sparsity between minutes 1 and 2 . Dashed line is best-fit line from linear regression, which showed a significant relationship with $r=0.67, P=0.0011$ (using the spatial information measure instead, $P=0.008$ ). a
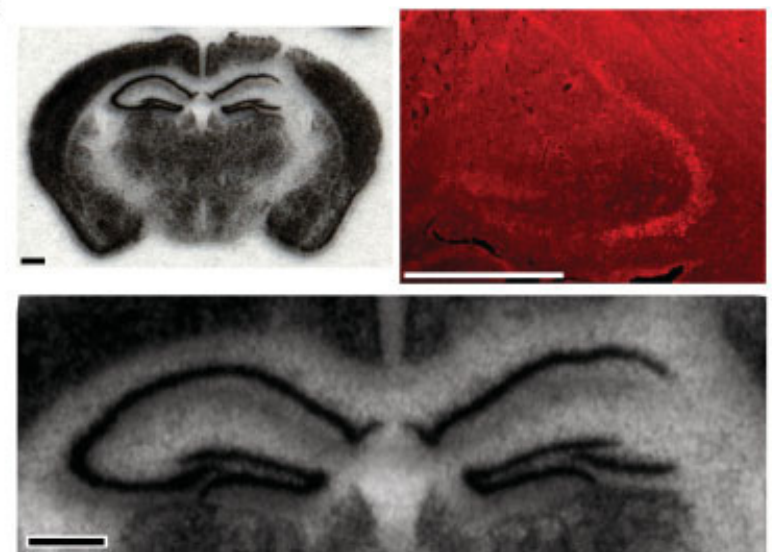

b

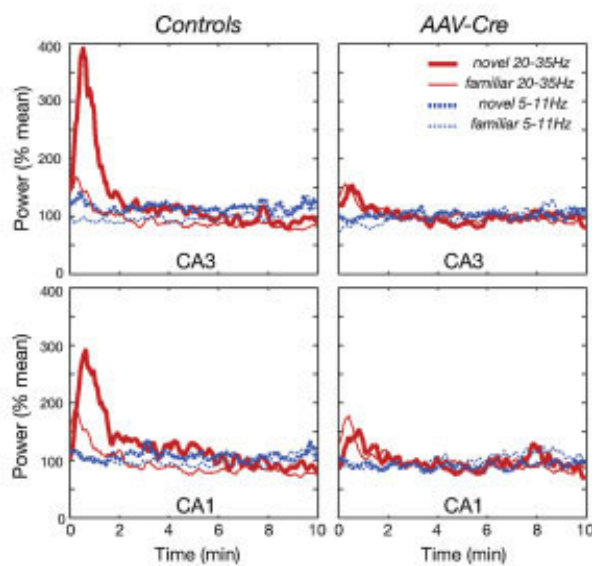

FIGURE 7. CA3 NMDA receptors are required for noveltyenhancement of beta 2 oscillations. a, (left and bottom) Coronal brain section with in situ hybridization for NR1 mRNA, for a mouse injected with AAV-Cre into right dorsal hippocampus. Excision of the NR1 gene was confined to the CA3 region in all included subjects. (top right) Corresponding immunolabeling of
Cre protein in infected CA3 cells in the same animal. Scale bars, $0.5 \mathrm{~mm}$. b, Time courses of beta2, theta power in control (left) and AAV-Cre treated (right) mice, ipsilateral to viral injection site. AAV-Cre abolishes the novelty-enhancement of beta2 oscillations, while the smaller increase in beta 2 seen in familiar environments remains. 
hand LFP traces of Figs. 4(a,b)]. Enhanced power in the beta2 band has been previously described in mutant mice lacking the HNK-1 sulfotransferase (Gurevicius et al., 2007), although novelty/familiarity differences were not tested. The enhanced beta 2 oscillations in those mice may reflect a deficit in forming stable, familiar environment representations, since the HNK-1 carbohydrate has been implicated in synaptic plasticity and learning (Bukalo et al., 2007; Morita et al., 2007). All the work presented here was performed in $f N R 1$ mice. These mice are generally considered to be normal unless the Cre protein is introduced, and have been used as the "control" mice in a number of behavioral and electrophysiological studies of hippocampus (Tsien et al., 1996a; Nakazawa et al., 2002, 2003). Nonetheless, this genetic modification makes it especially important to examine possible contributions of species and strain differences to novelty-induced beta 2 oscillations. For example, genetic background can have a strong influence over the incidence of pathological oscillations (i.e., seizures). Although often of considerable amplitude, we do not think it appropriate to classify our novelty-evoked beta2 oscillations as pathological, given that they did not disturb either the exploratory behavior of the mice (e.g., Supplementary Video Online) or the ongoing encoding of spatial representations.

The design of our behavioral experiments may also have contributed to our striking observations of beta2 oscillations. Few studies have investigated the early minutes of exploration of novel environments during which CA1 place fields develop, and most of those have looked at the incorporation of a novel zone into a previously explored environment (Wilson and McNaughton, 1993; Frank et al., 2004). By contrast, our novel environments were very distinct, incorporating novel shape, texture and odor. While we did not intentionally disorient the mice, the high walls of the chambers and central obstacles reduced their ability to localize themselves within a larger laboratory context, and to perceive the whole of the novel environment simultaneously. This latter point may bear upon one of our most striking findings-that beta2 power was weak or absent during the first lap, and became strong only as the mice began to encounter specific parts of the track for the second or third time. While we can currently only speculate why this might be so, we note that hippocampal representations are dependent upon the size of the encoded environment, among other factors (O'Keefe and Burgess, 1996). Until the mouse reencounters its maze entry point, the mouse lacks information critical to forming an appropriately scaled and integrated contextual representation.

If beta2 oscillations are involved in the encoding of novel environments, the delay that we observed before the onset of beta2 oscillations would suggest that such encoding is similarly delayed. Behavioral evidence that this is indeed the case is provided by experiments on contextual fear conditioning, for which the hippocampus is an essential structure. Exposing rats or mice to just a few seconds of an environment before giving a footshock does not result in robust contextual fear conditioning (the "immediate shock deficit"), while $\sim 30-60$ s of active exploration is typically sufficient (Fanselow, 2000; Frankland et al., 2004; McHugh and Tonegawa, 2007).
Although the tetrodes used here are not optimal for detailed exploration of the cellular/synaptic mechanisms responsible for hippocampal beta2, our observations are highly consistent with processes elucidated through in vitro studies. In hippocampal slices, oscillatory activity in the beta/gamma range can be readily produced by certain drugs, notably cholinergic agonists (Fisahn et al., 1998; Cobb and Davies, 2005; Arai and Natsume, 2006). These oscillations appear to be generated within CA3, require NMDA receptors for their initiation, and normal $\mathrm{GABA}_{\mathrm{A}}$ transmission for their maintenance (Shimono et al., 2000; Arai and Natsume, 2006). Fast-spiking GABAergic interneurons are particularly critical for these rhythms, due to their resonant membrane properties and the time constants of $\mathrm{GABA}_{\mathrm{A}}$ transmission (Pike et al., 2000; Bartos et al., 2007). In addition, the widespread connections of interneuron networks play a critical role in synchronizing oscillatory activity throughout hippocampus (Buzsáki, 2006). Altered NMDA function in interneuron populations with divergent connections may explain why we observed a substantial decrease in beta2 oscillations in both CA1 and CA3, following a viral manipulation that disrupted NMDA receptors in only a limited portion of the septo-temporal extent of CA3 ( 10-30\%; Rajji et al., 2006). The same viral manipulation, when given bilaterally, is sufficient to disrupt new learning (Rajji et al., 2006). Our observations suggest that, in addition to their better-characterized role gating calcium entry to affect local synaptic strength, CA3 NMDA receptors may contribute to learning via an influence over circuit- and systems-level dynamics.

Given the in vitro findings, an important question for future research is whether novelty-evoked beta2 oscillations also require altered cholinergic neuromodulation of hippocampal neurons. Acetylcholine (ACh) is provided to the hippocampus by neurons in the medial septum, and hippocampal ACh release is enhanced during exploration of novel environments (Giovannini et al., 2001). In turn, "long-range interneurons" in the hippocampus project to the septum (Toth et al., 1993), providing a pathway by which the hippocampus can dynamically regulate its own neuromodulation. Computational models (Meeter et al., 2004) have suggested that when placed in a novel environment, CA3 autoassociative networks fail to achieve pattern completion of a previously stored environmental representation; this leads to enhanced ACh release in hippocampus, which facilitates the reorganization of hippocampal representations (Hasselmo, 1999; Ikonen et al., 2002).

It is notoriously challenging to establish whether brain oscillations are simply an indicator of a dynamic network state, or play a causal role in brain function. However, it has been noted that a possible contribution of fast oscillations is in spike-timing dependent plasticity (Harris et al., 2003). Our observations suggest that during novelty-evoked beta2, synchronized networks of fast-spiking interneurons impose narrow windows in which pyramidal cells are able to fire, orchestrating them to fire together within the $\sim 10 \mu$ s range optimal for synaptic change (Bi and Poo, 1998). In this way, beta 2 oscillations may be both a discrete hippocampal functional state, and a mechanism for enabling plasticity during learning. 
METHODS

\section{Behavior, Viral Injections, and Electrophysiology}

All procedures were approved by the University of Michigan committees on Animal Use and Biosafety. Adult male fNRI mice (Tsien et al., 1996b), bred from a breeding pair generously provided by the Tonegawa laboratory, were housed on an 12:12 light:dark cycle and tested during the light phase. No food or water restriction was used. Each mouse was implanted with six tetrodes into dorsal hippocampus (as well as several skull screws for EEG recording), and in the same surgery some mice were additionally infused with AAV-Cre or AAV-LacZ using a picospritzer as described (Scammell et al., 2003). Tetrodes were progressively lowered into CA3 and CA1 pyramidal layers under electrophysiological guidance, and behavioral experiments began 8-14 days after surgery, to allow complete expression of the Cre transgene in the infected neurons (Scammell et al., 2003). (In one case without a viral injection, behavioral experiments were started 4 days after surgery). Tetrodes were not moved during the behavioral testing days. Single-unit spikes and LFPs were recorded using standard methods. For five animals, spikes were sampled at $300-6,000 \mathrm{~Hz}, 30,330$ samples/s and LFPs at $0.1-600 \mathrm{~Hz}, 1,500$ samples/s (software package Spike by Loren Frank). For the remaining animals, all wires were recorded wide-band $(1-9,000 \mathrm{~Hz}, 31,250$ samples/ s), then filtered and downsampled offline. All LFP signals were referenced to a skull screw on the midline slightly posterior to lambda. Raw LFPs are shown with positive polarity upwards. Up to 80 individual hippocampal neurons were recorded simultaneously and discriminated using manual clustering. Some cells (especially in CA3) that had low activity during exploration of the novel environment were detected because of their activity during sleep before and after exploration. Based upon location, firing pattern (phasic with complex-spike bursts vs. tonic), and waveform duration (Ranck, 1973; Csicsvari et al., 1998), the cell set included 31 CA3 pyramidal cells, 3 CA3 inhibitory interneurons, 21 CA2 pyramidal cells, 66 CA1 pyramidal cells, and 4 CA1 inhibitory interneurons. One tonic, wide-valley cell was unclassified. Position was monitored by overhead camera tracking (15-30 samples/s) of two infrared LEDs mounted on the animals' headstage. Recording locations were marked by passing current through the tetrode after recordings were completed, to create small lesions. For all viralinjected animals the extent of NR1 deletion was examined using in situ hybridization following standard protocols (Rajji et al., 2006). Cre protein expression was detected using rabbit anti-Cre antibody (Novagen \#69050-3) with goat antirabbit Cy3-conjugated secondary antibody (Jackson Immunoresearch \#111-165-144).

Neural activity was recorded in three different environments for each mouse. During the period of postsurgical recovery and tetrode adjustment, mice were habituated to a small square high-sided cardboard box $(20.5 \mathrm{~cm}$ on each side), and this square box was used as a holding chamber before and after each exposure to the test environments. The test environments were one circular $(34 \mathrm{~cm}$ diameter $)$ and one rectangular $(33 \times$ $27 \mathrm{~cm}$ ) environment, each with a tall internal object of the same shape serving to constrain the mice to a $\sim 6 \mathrm{~cm}$ wide track. The environments differed in floor texture (bare metal with sandpaper strips vs. fine wire mesh) and odor (odors used were cinnamon, cardamom, marjoram and nutmeg). The order of environments and odors was counterbalanced across animals. Mice typically ran a series of laps in one direction before changing direction. After $45 \mathrm{~min}$ the mice were removed and placed back in the holding chamber. The interval between being taken out of each environment and being placed in the next test environment was $2 \mathrm{~h}$ (Novel $\rightarrow$ Repeat 1; Repeat 2 $\rightarrow$ Novel) or overnight (Repeat $1 \rightarrow$ Repeat 2).

Seventeen experiments (mice) were attempted, of which eight were considered successful (3 no-virus, 2 AAV-LacZ, 3 AAVCre). Three mice died before the experiment was complete, for three animals the tetrode sites could not be determined through histological analysis, and one animal was not run in the test environments due to technical problems with the recording apparatus. Two additional AAV-Cre injected animals were excluded from statistical analysis due to an inability to determine the full extent of viral effect (although both showed suppression of novelty-evoked beta2). All included mice had tetrodes in both CA1 and CA3 pyramidal layers, except one AAVLacZ mouse (CA1 only), and one AAV-Cre mouse (CA1 electrode was in a deeper layer). Since no differences were observed between no-virus mice and control AAV-LacZ mice (both AAV$L a c Z$ mice showed the highest beta2 power in the novel environments), data from these groups were pooled to form a single "control" group $(n=5)$.

\section{Data Analysis and Statistics}

Mathematical analyses were performed using MATLAB. To generate moment-by-moment speeds for visual comparison to LFP rhythms, position values were smoothed with a $0.33 \mathrm{~s}$ moving average and speed calculated across a $0.2 \mathrm{~s}$ window around each time point. To determine prolonged epochs of active exploration vs. relative inactivity, position values were smoothed with a 20-s box filter and intervals were identified in which the speed remained below $5 \%$ of the environment diameter per second for a minimum duration of $20 \mathrm{~s}$. The fraction of time in the first $10 \mathrm{~min}$ of a recording session spent outside of those intervals was reported as proportion of time running. For the analysis of single neurons, we used just the first novel session (for each of the three no-virus mice), in order to avoid the possibility of counting the same neurons multiple times. To assess entrainment to beta2 oscillations, we first detected intervals of high beta2 power as described (Berke et al., 2004), within the $10 \mathrm{~min}$ following entry into a novel environment. Each spike that fell between two adjacent troughs of the $20-35 \mathrm{~Hz}$ filtered signal was assigned a corresponding phase (using $30^{\circ}$ bins); mean angle $(\Phi)$, vector length $(r)$ and significance of the resulting phase histogram was determined through calculation of Rayleigh's $Z$ (Zar, 1974). Estimation of 
theta entrainment was performed in the same manner with the 5-11 Hz filtered signal, using detected intervals in which 5-11 $\mathrm{Hz}$ power was more than double $2-4 \mathrm{~Hz}$ power, over the whole $\sim 1 \mathrm{~h}$ session. When comparing spatial specificity of beta2-entrained vs. nonentrained cells we limited the detection of beta 2 epochs to the first $90 \mathrm{~s}$, since we were concerned with beta 2 epochs that could be driving early changes in spatial specificity.

For the analysis of place-specific firing, we converted the two-dimensional positions on the tracks into one-dimensional angles from the maze center and split this angle into $N$ bins ( $N=50$ for the visualization of place maps as in Fig. 6a; $N=$ 10 for the calculation of spatial specificity). For a given minute, if $R_{\mathrm{i}}=$ firing rate of a neuron within a bin and $R=$ overall firing rate of that neuron, then:

Spatial information per spike (bits) $=$

$$
\begin{aligned}
& \sum_{i}\left(R_{i} / R\right) \log _{2}\left(R_{i} / R\right) / N \quad \text { (Skaggs et al., 1993) } \\
& \text { Sparsity }=\frac{\sum_{i}\left(R_{i} / N\right)^{2}}{\sum_{i}\left(R_{i}^{2} / N\right)} \quad \text { (Jung et al., 1994) }
\end{aligned}
$$

Sparsity is a measure of the distribution of activity over the available bins, with lower values indicating a less diffuse spike distribution; if activity is uniformly distributed over all bins, the information per spike $=0$ and the sparsity $=1$. Note that the minute-by-minute analysis of spatial specificity likely underestimates changes that occur very rapidly within the first minute.

Multitaper spectrograms (e.g., Fig. 1c) were generated using the Chronux library function mtspecgramc (www.chronux.org) on LFPs resampled to $256 \mathrm{~Hz}$, with a time window of $3 \mathrm{~s}$ and an increment of $1 \mathrm{~s}$. These parameters were also used to generate power values in 2-min blocks for statistical analysis, by summing the coefficients within the desired frequency band $(20-35$ or $5-11 \mathrm{~Hz})$ over the desired time range. In cases where there were multiple tetrode sites localized to a given hippocampal subregion, LFP power was averaged between these sites; for cases in which continuous data was obtained from all four tetrode wires, these were also averaged. The lap-by-lap power calculations were generated the same way, except that a time window of $1.5 \mathrm{~s}$ and an increment of $0.25 \mathrm{~s}$ were used. Scalograms were obtained by convolving the resampled LFP signal with complex Morlet wavelets (with six modes; Torrence and Compo, 1998). This wavelet analysis was also used to obtain moment-by-moment beta 2 and theta power values for Figure 3, by smoothing the coefficients for the corresponding scales with a 1-s box filter, and comparing to instantaneous speed values calculated as distance traveled over 1 s. Power spectral density plots were determined using Welch's method, with 1,024 frequencies in the $0-128 \mathrm{~Hz}$ range followed by smoothing with a four-point moving average.
While novelty-evoked beta2 was consistently observed in both CA3 and CA1 subregions, the relative power in the two regions varied between animals; this may reflect slight variation in tetrode positions, as oscillatory power can change rapidly with depth in the pyramidal layer (Buzsaki et al., 2003). We therefore normalized all beta 2 and theta values to the average power in the first $10 \mathrm{~min}$ across all test sessions (i.e., a grand mean). This is a conservative measure as it will tend to diminish differences between sessions. Similar or identical statistical differences between novel and familiar, and AAV-Cre and controls, were obtained using an alternative measure, $\log$ (beta2/theta ratio), but this measure was not used in the text as it does not allow us to directly contrast changes in beta2 with changes in theta.

The statistical analyses (run in SPSS14.0 for Windows) were designed to address several specific questions. To determine whether beta 2 power changed across sessions, and across timeblocks within sessions, we used a mixed-model ANOVA with a subject factor (MOUSE, "control" group only), and repeated measures factors of LOCATION (CA3 vs. CA1), TIMEBLOCK (0-2 min, 2-4 $\mathrm{min}, 4-6 \mathrm{~min}, 6-8 \mathrm{~min})$, and SESSION (seven levels as shown in Fig. 2a). The analysis indicated significant main effects of TIMEBLOCK $(F=24.54, P<$ $0.001)$ and SESSION $(F=14.11, P<0.001)$ but not of LOCATION $(F=0.00, P>0.1)$, nor were any interactions significant. Given that session affected beta 2 power, we proceeded with planned comparisons contrasting the two novel sessions with the four familiar sessions, as indicated in the main text. To determine whether our AAV-Cre manipulation affected beta 2 power in the first $2 \mathrm{~min}$ of novel sessions, we ran another mixed model ANOVA, again with a subject factor (MOUSE, all mice included), and factors LOCATION, NOVELTY (novel vs. familiar sessions) and VIRUS (pooled controls vs. AAV-Cre). The results showed a significant VIRUS $\times$ NOVELTY interaction $(F=18.27, P<0.001)$, and no further interaction with LOCATION. By inspection of Figure 7 , these results were interpreted as an AAV-Cre suppression of novelty-evoked beta2 in both CA3 and CA1.

\section{Acknowledgments}

We thank Howard Eichenbaum, in whose laboratory some of these data were collected, Lotus McDougal for technical assistance, John Jackson for statistical advice, and Loren Frank, Terry Robinson, and Colin Stoetzner for comments on prior versions of the manuscript. Murat Okatan and Steven Kunec contributed MATLAB routines.

\section{REFERENCES}

Adrian ED. 1942. Olfactory reactions in the brain of the hedgehog. J Physiol 100:459-473.

Arai J, Natsume K. 2006. The properties of carbachol-induced beta oscillation in rat hippocampal slices. Neurosci Res 54:95-103.

Bartos M, Vida I, Jonas P. 2007. Synaptic mechanisms of synchronized gamma oscillations in inhibitory interneuron networks. Nat Rev Neurosci 8:45-56. 
Berke JD, Okatan M, Skurski J, Eichenbaum HB. 2004. Oscillatory entrainment of striatal neurons in freely moving rats. Neuron 43:883-896.

Bi GQ, Poo MM. 1998. Synaptic modifications in cultured hippocampal neurons: Dependence on spike timing, synaptic strength, and postsynaptic cell type. J Neurosci 18:10464-10472.

Bukalo O, Schachner M, Dityatev A. 2007. Hippocampal metaplasticity induced by deficiency in the extracellular matrix glycoprotein tenascin-R. J Neurosci 27:6019-6028.

Buzsáki G. 2006. Rhythms of the Brain. New York: Oxford University Press.

Buzsaki G, Buhl DL, Harris KD, Csicsvari J, Czeh B, Morozov A. 2003. Hippocampal network patterns of activity in the mouse. Neuroscience 116:201-211.

Cobb SR, Davies CH. 2005. Cholinergic modulation of hippocampal cells and circuits. J Physiol 562:81-88.

Csicsvari J, Hirase H, Czurko A, Buzsaki G. 1998. Reliability and state dependence of pyramidal cell-interneuron synapses in the hippocampus: An ensemble approach in the behaving rat. Neuron 21:179-189.

Csicsvari J, Jamieson B, Wise KD, Buzsaki G. 2003. Mechanisms of gamma oscillations in the hippocampus of the behaving rat. Neuron 37:311-322.

Diba K, Buzsaki G. 2007. Forward and reverse hippocampal place-cell sequences during ripples. Nat Neurosci 10:1241-1242.

Eichenbaum H, Cohen NJ. 2001. From Conditioning to Conscious Recollection: Memory Systems of the Brain. New York: Oxford University Press.

Fanselow MS. 2000. Contextual fear, gestalt memories, and the hippocampus. Behav Brain Res 110:73-81.

Fisahn A, Pike FG, Buhl EH, Paulsen O. 1998. Cholinergic induction of network oscillations at $40 \mathrm{~Hz}$ in the hippocampus in vitro. Nature 394:186-189.

Foster DJ, Wilson MA. 2006. Reverse replay of behavioural sequences in hippocampal place cells during the awake state. Nature 440:680-683.

Frank LM, Stanley GB, Brown EN. 2004. Hippocampal plasticity across multiple days of exposure to novel environments. J Neurosci 24:7681-7689.

Frankland PW, Josselyn SA, Anagnostaras SG, Kogan JH, Takahashi E, Silva AJ. 2004. Consolidation of CS, US representations in associative fear conditioning. Hippocampus 14:557-569.

Fuchs EC, Zivkovic AR, Cunningham MO, Middleton S, Lebeau FE, Bannerman DM, Rozov A, Whittington MA, Traub RD, Rawlins JN, Monyer H. 2007. Recruitment of parvalbumin-positive interneurons determines hippocampal function and associated behavior. Neuron 53:591-604.

Giovannini MG, Rakovska A, Benton RS, Pazzagli M, Bianchi L, Pepeu G. 2001. Effects of novelty and habituation on acetylcholine, GABA, and glutamate release from the frontal cortex and hippocampus of freely moving rats. Neuroscience 106:43-53.

Grossberg S. 1999. The link between brain learning, attention, and consciousness. Conscious Cogn 8:1-44.

Gurevicius K, Gureviciene I, Sivukhina E, Irintchev A, Schachner M, Tanila H. 2007. Increased hippocampal and cortical beta oscillations in mice deficient for the HNK-1 sulfotransferase. Mol Cell Neurosci 34:189-198.

Harris KD, Csicsvari J, Hirase H, Dragoi G, Buzsaki G. 2003. Organization of cell assemblies in the hippocampus. Nature 424:552-556.

Hasselmo ME. 1999. Neuromodulation: Acetylcholine and memory consolidation. Trends Cogn Sci 3:351-359.

Hasselmo ME, Bodelon C, Wyble BP. 2002. A proposed function for hippocampal theta rhythm: Separate phases of encoding and retrieval enhance reversal of prior learning. Neural Comput 14:793-817.

Ikonen S, McMahan R, Gallagher M, Eichenbaum H, Tanila H. 2002. Cholinergic system regulation of spatial representation by the hippocampus. Hippocampus 12:386-397.
Jung MW, Wiener SI, McNaughton BL. 1994. Comparison of spatial firing characteristics of units in dorsal and ventral hippocampus of the rat. J Neurosci 14:7347-7356.

Knight R. 1996. Contribution of human hippocampal region to novelty detection. Nature 383:256-259.

Lee I, Kesner RP. 2002. Differential contribution of NMDA receptors in hippocampal subregions to spatial working memory. Nat Neurosci 5:162-168.

McHugh TJ, Tonegawa S. 2007. Spatial exploration is required for the formation of contextual fear memory. Behav Neurosci 121:335339.

Meeter M, Murre JM, Talamini LM. 2004. Mode shifting between storage and recall based on novelty detection in oscillating hippocampal circuits. Hippocampus 14:722-741.

Morita I, Kizuka Y, Kakuda S, Oka S. 2007. Expression and function of the HNK-1 carbohydrate. J Biochem. Advance Access published on November 20, 2007; DOI: 10.1093/jb/mvm221.

Nakazawa K, Quirk MC, Chitwood RA, Watanabe M, Yeckel MF, Sun LD, Kato A, Carr CA, Johnston D, Wilson MA, Tonegawa S. 2002. Requirement for hippocampal CA3 NMDA receptors in associative memory recall. Science 297:211-218.

Nakazawa K, Sun LD, Quirk MC, Rondi-Reig L, Wilson MA, Tonegawa S. 2003. Hippocampal CA3 NMDA receptors are crucial for memory acquisition of one-time experience. Neuron 38:305-315.

O'Keefe J, Burgess N. 1996. Geometric determinants of the place fields of hippocampal neurons. Nature 381:425-428.

Pike FG, Goddard RS, Suckling JM, Ganter P, Kasthuri N, Paulsen O. 2000. Distinct frequency preferences of different types of rat hippocampal neurones in response to oscillatory input currents. J Physiol 529:205-213.

Rajji T, Chapman D, Eichenbaum H, Greene R. 2006. The role of CA3 hippocampal NMDA receptors in paired associate learning. J Neurosci 26:908-915.

Ranck JB Jr. 1973. Studies on single neurons in dorsal hippocampal formation and septum in unrestrained rats. I. Behavioral correlates and firing repertoires. Exp Neurol 41:461-531.

Ravel N, Chabaud P, Martin C, Gaveau V, Hugues E, Tallon-Baudry C, Bertrand O, Gervais R. 2003. Olfactory learning modifies the expression of odour-induced oscillatory responses in the gamma $(60-90 \mathrm{~Hz})$ and beta $(15-40 \mathrm{~Hz})$ bands in the rat olfactory bulb. Eur J Neurosci 17:350-358.

Scammell TE, Arrigoni E, Thompson MA, Ronan PJ, Saper CB, Greene RW. 2003. Focal deletion of the adenosine A1 receptor in adult mice using an adeno-associated viral vector. J Neurosci 23: 5762-5770.

Shimono K, Brucher F, Granger R, Lynch G, Taketani M. 2000. Origins and distribution of cholinergically induced beta rhythms in hippocampal slices. J Neurosci 20:8462-8473.

Skaggs WE, McNaughton BL, Gothard KM, Markus EJ. 1993. An information-theoretic approach to deciphering the hippocampal code. Adv Neuro Info Proc Syst 5:1030-1037.

Thompson LT, Best PJ. 1990. Long-term stability of the place-field activity of single units recorded from the dorsal hippocampus of freely behaving rats. Brain Res 509:299-308.

Torrence C, Compo GP. 1998. A practical guide to wavelet analysis. Bull Am Meteorol Sco 79:61-78.

Toth K, Borhegyi Z, Freund TF. 1993. Postsynaptic targets of GABAergic hippocampal neurons in the medial septum-diagonal band of broca complex. J Neurosci 13:3712-3724.

Tsien JZ, Chen DF, Gerber D, Tom C, Mercer EH, Anderson DJ, Mayford M, Kandel ER, Tonegawa S. 1996a. Subregion- and cell type-restricted gene knockout in mouse brain. Cell 87:1317-1326.

Tsien JZ, Huerta PT, Tonegawa S. 1996b. The essential role of hippocampal CA1 NMDA receptor-dependent synaptic plasticity in spatial memory. Cell 87:1327-1338.

Wilson MA, McNaughton BL. 1993. Dynamics of the hippocampal ensemble code for space. Science 261:1055-1058. 